\title{
Camera Based Artificial Intelligence for A Smart Vehicle Braking System
}

\author{
*Sonki Prasetya ${ }^{1,3 *}$, Hasvienda M. Ridlwan ${ }^{3}$, Hendri DS Budiono ${ }^{2}$, Ario Sunar Bhaskoro ${ }^{2}$, \\ Agung Shamsuddin ${ }^{2}$, Mohammad Adhitya ${ }^{2}$, Danardono A Sumarsono ${ }^{3}$ \\ ${ }^{1}$ Student of Mechanical Engineering Department, Engineering Faculty of Universitas Indonesia, Depok 16424, \\ Indonesia \\ ${ }^{2}$ Mechanical Engineering Department, Engineering Faculty of Universitas Indonesia, Depok 16424, Indonesia \\ ${ }^{3}$ Mechanical Engineering Politeknik Negeri Jakarta, Depok 16424, Indonesia \\ sonki.prasetya@mesin.pnj.ac.id
}

\begin{abstract}
A braking system is an essential value in a vehicle particularly for a safety precaution. The higher number rate of traffic accidents mostly in Indonesia shows that the dominant cause of accidents is due to the human factor. The physical condition such as tired or sleepy during driving is the primary problem according to the survey. In order to assist a person when driving a vehicle, an artificial intelligence method is necessary to be integrated to ensure the safety of surrounding people inside and outside the vehicle. This study focuses on implementing the method of identifying object and distance to provide an indicator for braking action. Images from a stereo camera is processed by a neural network technique via a mini computer to classify as well as the distance of objects. Furthermore, selection of priorities are done to obtain the intensity of braking action. The result shows that process of classification and measurement requires period around 200 ms. Furthermore, braking action done by fuzzy controller sub-system shows that the intensity has smoother signal with the object distance variation compare to the direct method. Objects are firstly identified by the presence of a stereo camera, later on the decision of braking intensity is generated by two processing unit namely conventional and fuzzy unit. This is achieved by processing the data saved from the object detection using two system via MATLAB software. The object identification result, distance measurement and the period of object detection is presented. Moreover, the response of braking intensity using data is processed with both conventional and fuzzy unit systems are also presented. he implementation of this study is for the heavy vehicle such buses or trucks that requires higher safety during the journey.
\end{abstract}

Key words : Braking system, Fuzzy unit, Neural network, Stereo camera, MATLAB, Artificial intelligence camera

\section{INTRODUCTION}

A vehicle used for transporting human from one to another place has been developed rapidly during decades.
Commercial 4 wheels vehicle data show that over 80 million units are sold in 2018 [1]. Naturally, there are effects due to the higher number of owned vehicles. One of the negative impact of vehicle utilizations is the accident. The World Health Organization recorded that more than 1.3 million people died due to the vehicle accident annually [2]. In a capital city namely Jakarta, a highway accident data show that most of accident caused by human such as tired condition like sleepy [3]. Therefore, a device to assist a person when driving is necessary to avoid this problem. Moreover, this intelligent braking assistance for driver can be developed as the foundation for an autonomous vehicle.

The commercial braking system for vehicles has several types. Friction is the most applied one. Hydraulic actuators normally found in light weighted vehicles for creating friction movement mechanically from the pushed brake pedal. Furthermore, for heavy weighted vehicles use pneumatic actuators. This system is a part of study of utilizing the electrical signal to move the braking actuator.

There has been researches for advanced vehicles harnessing an intelligent methods. Computational perception using visual and laser scanner is developed by Martin [4]. Furthermore, braking system by vision using Support Vector Machine is done by Wang [5]. The low cost intelligent system for vehicle was the objective of Heimberger to develop three dimensional reconstruction by vision for auto parking [6].

The aim of this study is to generate an electrical signal based on the intensity for braking activation. Using two indicators namely object classification and distance, the intensity of braking is decided. The result is then transferred to the signal conditioning for the input of the actuators.

\section{METHODOLOGY}

This paper covers two main stages of the system. Those two stages are described as the object detection and the braking decision. 


\subsection{Object Detection}

The first stage depends on the stereo camera principle as the input of the system. It uses two cameras namely left and right eyes. The concept uses the images captured by human eyes. Eyes either left or right retina send an image to the brain that formed a triangle position with an object point. Therefore, a triangulation method is utilized to determine the distance of an object in relation to the sensors (both cameras) [7]. A 3D image can be formed with a stereo reconstruction. The stages of the reconstruction are calibration, rectification and estimation.

The first stage requires focal length, point coordinates, radial and tangential distortion factor. ZED SDK (type of the camera) has the factory calibration data (self-calibration). Moreover, the second stage process of the camera is also self-provided by the manufacturer. Therefore, calibration and rectification is not required to be programmed when used. However, the depth estimation is derived by triangulation from the geometric model of non-distorted rectified camera. The calculation of the depth $(Z))$ is described as follow [8].

$$
Z=f \frac{b}{d}
$$

Where $d$ is disparity, $f$ is the focal length of the camera and is the baseline distance between the center of camera respectively. The disparity consist of the subtraction image positions (left and right) related to the center of the image frame in the $\mathrm{x}$-coordinate. Figure 1 depicts the illustration of the stereo camera arrangement.

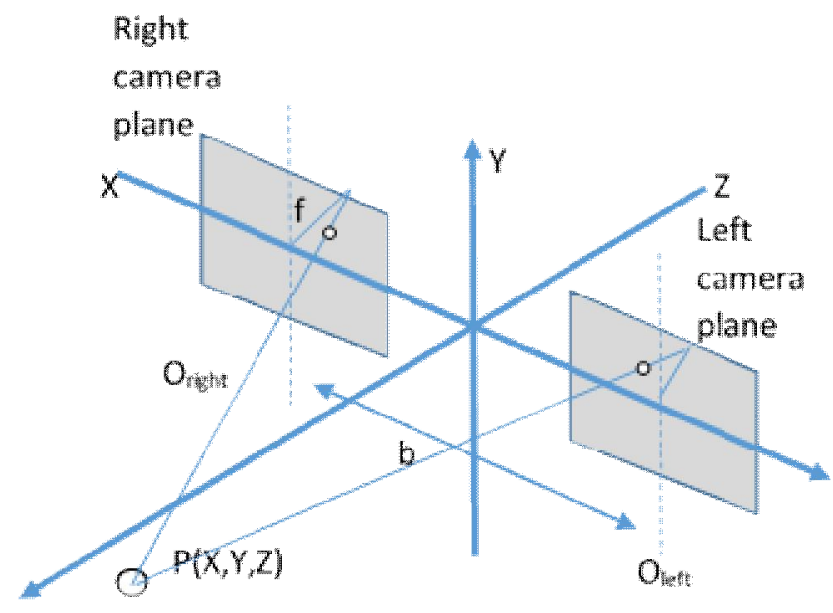

Figure 1: Stereo Camera an arrangement [8]

Object identification is derived using an intelligent method called Convolutional Neural Network (CNN). It is a special type of feed forward Neural Network method inspired by Brain part of Visual Cortex work. It uses neurons principle in order to classify an input. The weighting factors are generated from the given input to link the relationship between neurons. However, a training process is required to find the reference weights. Therefore, those values can be compared to others when we need to classify an object. The implementations of $\mathrm{CNN}$ can be found in several researches for instance for a facial detection [9], finding the data of the camera source [10], and also an Apps for Chinese menu uses this method [11]. The CNN Commonly it consist of several block system namely Convolution layer, Rectified Linear Unit (ReLU), Pooling and Fully Connected layer. Layer play important part in Neural Network. Numbers of layer follow the rule:

$$
a_{i, j}=f\left(\sum_{m=0}^{M} \sum_{n=0}^{N} w_{m, n} x_{i+m, j+n}+w_{b}\right)
$$

where a_(i,j) is pixel in it row and the column, $x_{-}(i, j)$ is the pixel of point $i$ and $j$ of input image, $w_{-}(m, n)$ is the weight of filter position, $\mathrm{w}_{-} \mathrm{b}$ is filter bias and $\mathrm{f}$ is the function respectively. Value of $\mathrm{w}_{-}(\mathrm{m}, \mathrm{n})$ and $\mathrm{w}$ _bare the parameters obtained from learning process and optimization during learning. Meanwhile size of the kernel (smallest matrix of an image) is $\mathrm{MxN}$.

Stages and the investigation scheme are shown in Figure 2. The complete setup consists of a stereo camera, a mini computer for processing the image and provide the decision in terms of signal electrical level. This signal is then proceed to the signal conditioning and finally transferred to the actuator for creating braking movement. However, this paper covers only in the generating signal part as shown inside of the dotted curve.

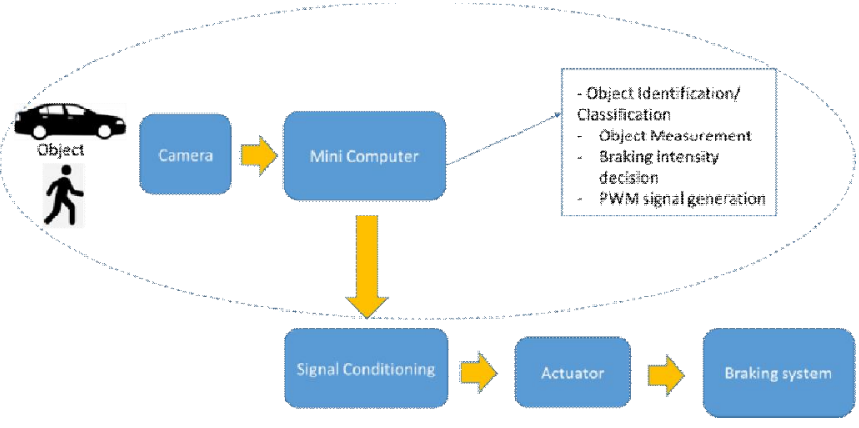

Figure 2: Scheme of the investigation

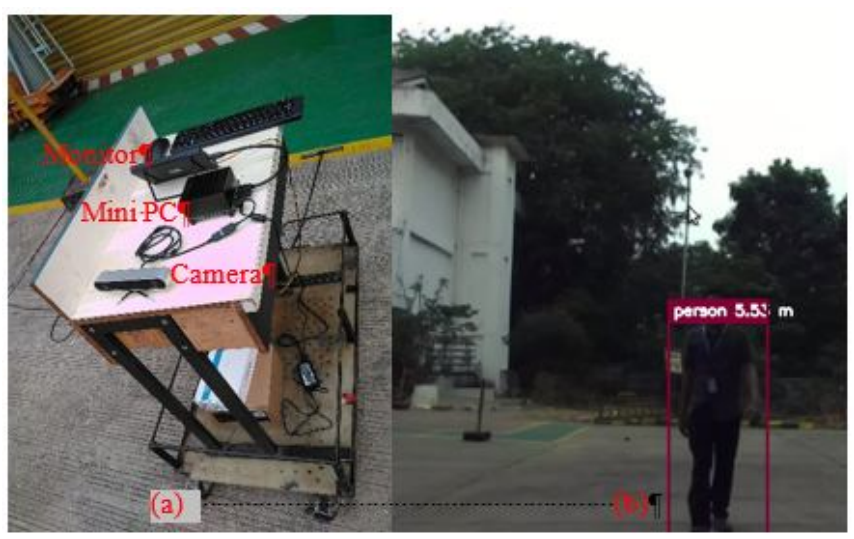

Figure 3: Object detection experiment 
Figure 3 is the experiment of object detection setup. The left (a) picture is the detection system that consists of the 3D camera connected with the mini PC with the monitor the monitor to display the images camera captured and also the result of detection. The detection data is saved into the $\mathrm{PC}$ to be processed for obtaining the braking action intensity value. Meanwhile, the right image (b) is the object detection result in the monitor display. It presents the image with a box named the classified object as well as the distance.

After the object classification/ detection. The second stage is to decide the braking force. In order to test the brake, the test rig for the heavy vehicle braking is built with a quarter system (only one wheel). However, this paper only describes the braking intensity generation decided by the data taken from the object. The experiment is performed in an open area to simulate the real condition of the vehicle.

\subsection{Braking Decision}

In order to generate the braking action signal, two methods are considered. The first one is directly converted into braking decision with range intensity into four stages from 0 to 20 level. The other one is using fuzzy to decide the braking action.

Fuzzy logic is a method approach by imitating the human way of decision. This method has been implemented to many sectors namely medical [12], farming [13] and also for detecting object using a stereo camera [14]. Furthermore, the researchers also used fuzzy method for electric vehicle applications for instance in energy reduction of vehicle consumption [15], regenerative braking [16], autonomous controlling vehicle in roundabout [17], parking lot [18], improvement of visual detection for pedestrian crossing [19]. This paper utilizes fuzzy system built via MATLAB toolbox. Therefore, several steps are done as mentioned. In order to create the fuzzy system, there are several segments to be considered. At the beginning, linguistic variables of input and output are defined. The input is organized into three classification of distance namely close, medium and far object range with respect to the camera. Furthermore, the output is ordered into three categories as well namely no-brake, medium-brake and hard-brake. Those groups represent the intensity of the braking action. The linguistic input and output can be presented as below.

The linguistic value of input variable universe

Person distance $(t)=($ far, medium, close $)$ with universe of discourse $[0,25]$

This means that the response observed of the braking is from distance below $25 \mathrm{~m}$ in front of the vehicle.

The linguistic value of output variable universe

Braking intensity $(\mathrm{t})=($ no brake, medium brake, hard-brake $)$ with interval $[0,20]$

This means that considered maximum intensity level of the braking signal is 20 .
Later on, the membership function is constructed in in Figure 4. Three input categories are presented in curves with created the linguistic of the input and output variable universes. Both input and output use triangular function shape for this study.
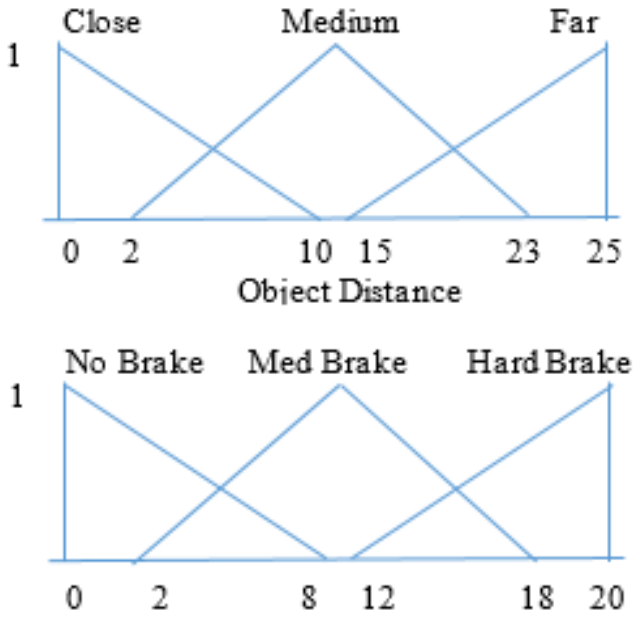

Figure 4 : Membership function

The knowledge base rules is made to match the relationship between input and the desired output. This can be derived using a look up table to consider the pairs of input and output.

- If (input 1 is close) then (output1 is hard brake)

- If (input 1 is medium) then (output1 is med brake)

- If (input 1 is far) then (output1 is no_brake)

De-fuzzification uses centroid method as seen as in Figure 5 with an example of input value. If a value of an input appears, it will be projected to the divided input of membership function at the horizontal coordinate plane (this study uses three curves as the input). Afterward, a vertical line is drawn to the curves. Therefore, it could intersects to more than one curve. The points of the curve that crossed by the vertical line are then projected to the output curves. The areas of the output are then added. Furthermore, the middle point of those areas is the result value.
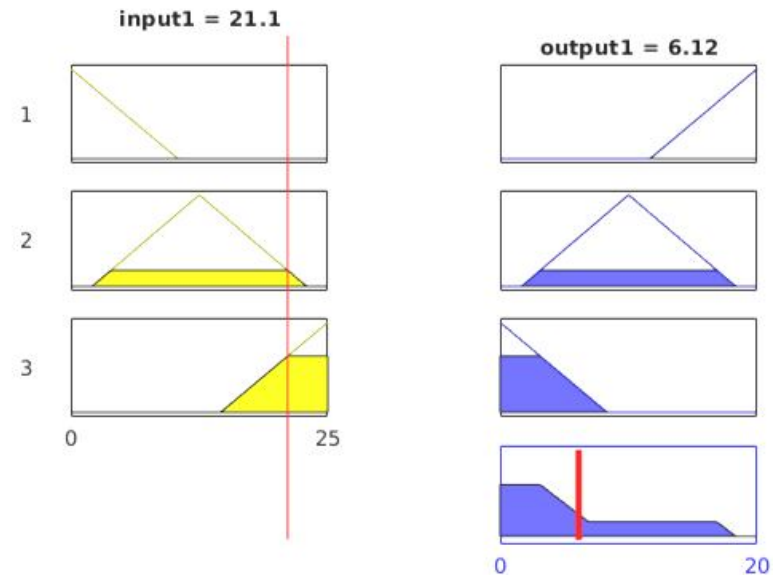

Figure 5 : Defuzzification result 


\section{RESULTS AND DISCUSSION}

The outcomes are presented in two stages as well. The first stage is to generate data of classifying object. The detected objects are used as the input data for the next stage. At the beginning, a partial test is done to ensure the machine works properly. The ability to classify objects, the object accuracies and also period of detections are the target. The accuracy is a parameter of the matching weighting values of the object sample with the captured image from the camera. Those outcomes are captured and presented in Table 1.

Table 1 : Accuracy and detection period

\begin{tabular}{cccc}
\hline No & Object & $\begin{array}{c}\text { Accuracy } \\
(\boldsymbol{\%})\end{array}$ & Duration (ms) \\
\hline 1 & Person 1 & 98 & 210 \\
2 & Person 2 & 97 & 190 \\
3 & Person 3 & 96 & 230 \\
4 & Person 4 & 99 & 220 \\
\hline
\end{tabular}

The accuracy shows that the object can be clearly distinguished more than $90 \%$. Furthermore, the period to predict the object is around $200 \mathrm{~ms}$.

Another data for the partial test is taken to find the error of the measured distance as seen on Table 2. The result shows that the maximum error of the detection is $2 \%$. The errors found are considered sufficient to be utilized for the vehicle detection object application.

Table 2 : Distance measurement comparison

\begin{tabular}{ccccc}
\hline No & Object & $\begin{array}{c}\text { Accuracy } \\
\text { ref }(\mathbf{m})\end{array}$ & $\begin{array}{c}\text { Duration } \\
\text { detected } \\
(\mathbf{m s})\end{array}$ & $\begin{array}{c}\text { Error } \\
(\%)\end{array}$ \\
\hline 1 & Person 1 & 10 & 10.15 & 1.5 \\
2 & Person 2 & 12 & 11.9 & 0.83 \\
3 & Person 3 & 17 & 17.1 & 0.59 \\
4 & Person 4 & 20 & 19.6 & 2 \\
\hline
\end{tabular}

The second stage called the braking decision is divided into two results namely direct step and fuzzy method. Input from the dataset of object movements is transferred to the MATLAB environment. The MATLAB Simulink blocks have been created with two processes (the direct conversion and the fuzzy braking). Those two result is presented in parallel to observe the difference between them (Figure 6).

The first figure with the green curve on the top is the input of object distances, the middle graph with the red curve is the direct braking action, and the last diagram with the blue curve is the fuzzy braking action. The result shows that the fuzzy method is more delicate result compare to the direct method. It is beneficial for vehicle implementation particularly regarding the people inside of the vehicle. The braking action creates disturbance to the object inside the vehicle. Therefore, smooth movement is preferable as it is more comfortable for the driver or the passengers.

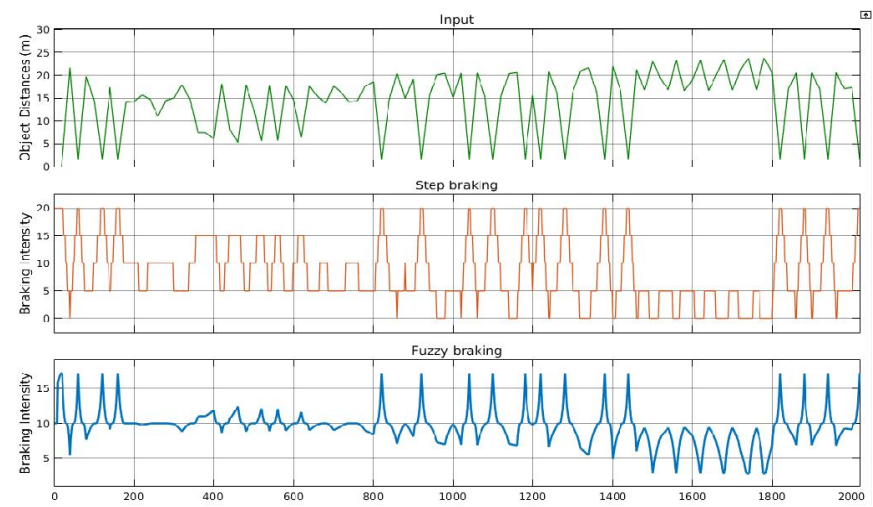

Figure 6 : Braking action with object movements

\section{CONCLUSION}

The summary of this paper is presented as follow: Object detection requires a period of recognition of around 200ms. The outcomes show that the object can be distinguished with more than $90 \%$ accuracy.

Error for distance measurement are vary with the maximum value of $2 \%$ is adequate for this application.

Fuzzy decision creates the signal results are more delicate compare to the direct method, this will affect to the comfort of the vehicle's driver/passengers.

For further development and system integration, the works of other researchers [20], [21] can be considered.

\section{ACKNOWLEDGEMENT}

The writers would like to thank the support of Penelitian Terapan Unggulan Perguruan Tinggi (PTUPT DIKTI) program for financing this work.

\section{REFERENCES}

1. H. Bekker. International: Worldwide Car Sales and Global Market Analysis Retrieved March, 2019.

2. World Health Organization (WHO). Global Status Report on Road Safety 2015: World Health Organization, 2015.

3. R. A. Pitoko. Traffic accidents in jagorawi toll road 13 cases per month. Retrieved January, 2019.

4. D. Martin, F. Garcia, B. Musleh, D. Olmeda, G. Pelaez, P. Marin, J. M. Armingol. IVVI 2.0: An Intelliegent Vehicle based on Computational Perception, Expert Systems with Applications Elsevier, vol. 41, no. 17, pp. 7927-7944, 2014.

5. X. Wang, J. Tang, J. Niu, and X. Zhao. Vision based Two step Brake Detection Method for Vehicle Collision Avoidance, Neuro Computing Elsevier, vol. 173, pp. 450-461, 2016. 
6. M. Heimberger, J. Horgan, C. Hughes, C., J. M. Donald, S. Yogamani. Computer Vision in Automated Parking Systems: Design Implementation and Challenges, Image and Vision Computing Elsevier, vol. 68, pp. 88-101, 2017.

7. R. Szeliski. Computer Vision: Algorithms and Applications: Springer Science \& Business Media, 2010.

8. Z. Hu, F. Lamosa, and K. Uchimura. A Compete U-V-Disparity Study for Stereovision Based 3D Driving Environment Analysis, in International Conference on 3-D Digital Imaging and Modeling (2DIM'05, pp. 204-211, 2005.

9. A. Jalali, R. Mallipeddi, and M. Lee. Sensitive deep convolutional neural network for face recognition at large standoffs with small dataset, Expert Systems with Applications, vol. 87, pp. 304-315, 2017 doi: https://doi.org/10.1016/j.eswa.2017.06.025

10. N. Huang, J. He, N. Zhu, X. Xuan, G. Liu, and C. Chang. Identification of the source camera of images based on convolutional neural network, Digital Investigation, vol. 26, pp. 72-80, 2018.

doi: https://doi.org/10.1016/j.diin.2018.08.001

11. M. C. Lee, S. Y. Chiu, and J. W. Chang. A Deep Convolutional Neural Network based Chinese Menu Recognition App, Information Processing Letters, 128, 14-20, and 2017. doi: https://doi.org/10.1016/j.ipl.2017.07.010

12. H. Ahmadi, M. Gholamzadeh, L. Shahmoradi, M. Nilashi, and P. Rashvand. Diseases diagnosis using fuzzy logic methods: A systematic and meta-analysis review, Computer Methods and Programs in Biomedicine, vol. 161, pp. 145-172, 2018. doi: https://doi.org/10.1016/j.cmpb.2018.04.013

13. M. S. Munir, I. S. Bajwa, and S. M. Cheema. An intelligent and secure smart watering system using fuzzy logic and blockchain, Computers \& Electrical Engineering, vol. 77, pp. 109-119, 2019. doi: https://doi.org/10.1016/j.compeleceng.2019.05.006

14. C. F. Juang, G. C. Chen, C. W. Liang, and D. Lee, D. Stereo-camera-based object detection using fuzzy color histograms and a fuzzy classifier with depth and shape estimations, Applied Soft Computing, vol. 46, pp. 753-766, 2016.

doi: https://doi.org/10.1016/j.asoc.2015.10.025

15. O. Kraa, M. Becherif, M. Y. Ayad, R. Saadi, M. Bahri, A. Aboubou, and I. Tegani. A Novel Adaptive Operation Mode based on Fuzzy Logic Control of Electrical Vehicle, Energy Procedia, vol. 50, pp. 194-201, 2014. doi: https://doi.org/10.1016/j.egypro.2014.06.024

16. R. Maia, M. Silva, R. Araújo, and U. Nunes. Electrical vehicle modeling: A fuzzy logic model for regenerative braking, Expert Systems with Applications, vol. 42, no. 22, pp. 8504-8519, 2015. doi: https://doi.org/10.1016/j.eswa.2015.07.006

17. J. P. Rastelli, M. S. Peñas. Fuzzy logic steering control of autonomous vehicles inside roundabouts, Applied Soft Computing, vol. 35, pp. 662-669, 2015. doi: https://doi.org/10.1016/j.asoc.2015.06.030

18. S. Faddel, A. T. Al-Awami, M. A. Abido. Fuzzy Optimization for the Operation of Electric Vehicle Parking Lots, Electric Power Systems Research, vol. 145, pp. 166-174, 2017. doi: https://doi.org/10.1016/j.epsr.2017.01.008

19. J. Albusac, D. Vallejo, J. J. Castro-Schez, and C. Gzlez-Morcillo. An expert fuzzy system for improving safety on pedestrian crossings by means of visual feedback, Control Engineering Practice, vol. 75, pp. 38-54, 2018. doi: https://doi.org/10.1016/j.conengprac.2018.03.008

20. Sharvani, C., Praveen Kumar, D., Bhavya, P., Sri Vaishnavi, P. Real time video enhancement using camera response model, International Journal of Emerging Trends in Engineering Research 8(5),47, pp. 1780-1784, 2020. https://doi.org/10.30534/ijeter/2020/47852020

21. Tran, T.M., Ta, K.D., Hoang, M., (...), Nguyen, N.D., Pham, G.N. A study on determination of simple objects volume using ZED stereo camera based on 3D-points and segmentation images, International Journal of Emerging Trends in Engineering Research 8(5),85, pp. 1990-1995, 2020. https://doi.org/10.30534/ijeter/2020/85852020 